\title{
A fully coupled implementation of hydrogen embrittlement in $\mathrm{FE}$ analysis
}

\author{
Giorgia Gobbi ${ }^{1}$, Chiara Colombo, Stefano Miccoli, Laura Vergani \\ Politecnico di Milano, Department of Mechanical Engineering, \\ Via G. La Masa 1, 20156 Milano, Italy
}

\section{Manuscript Info}

[April 29, 2019] This is an author generated postprint of the article:

"G. Gobbi, C. Colombo, S. Miccoli, L. Vergani, A fully coupled implementation of hydrogen embrittlement in FE analysis, Advances in Engineering Software, Volume 135, 2019, 102673, IssN 0965-9978, DOI 10.1016/j.advengsoft.2019.04.004,"

The version of record is available at https://doi.org/10.1016/j .advengsoft. 2019.04.004

The software described in this paper is available at https://doi.org/10.5281/zenodo.1478086

(c)2019. This manuscript version is made available under the Creative Commons Attribution-NonCommercialNoDerivatives 4.0 International License. [G. Er.Nc-ND

\begin{abstract}
Proper understanding of hydrogen embrittlement in steel is of paramount importance in several engineering applications, e.g. oil \& gas and hydrogen storage \& transport. This phenomenon can be modelled by means of a mass diffusion analysis driven by mechanical fields, i.e. hydrostatic stress gradient and plastic strain. Since the mechanical response depends on the hydrogen content itself, continuum mechanics and mass diffusion equations are fully coupled.

Accordingly in this paper a fully coupled-cohesive zone implementation is presented for the Abaqus Finite Element code, adopting the coupled thermal-stress analysis and the analogy between mass diffusion and heat transfer. The implementation requires extensive use of FORTRAN user subroutines and common blocks to share data, plus some auxiliary Python scripts.

With the aim to provide a practical example to the use of the code, a FE model reproducing a fracture toughness test of $\mathrm{C}(\mathrm{T})$ specimen charged with atomic hydrogen is described. Moreover, a sensitivity analysis of the model shows the capability of the developed numerical tool in predicting hydrogen embrittlement.

The code developed in this paper is open source under a permissive free software license.

Keywords: Hydrogen embrittlement, Finite element method, Cohesive elements, Coupled analysis
\end{abstract}

\footnotetext{
${ }^{1}$ Currently CERN, Engineering Department, Geneva Switzerland.
} 


\section{List of symbols}

$\bar{a}$

$A_{r}$

C

$C_{L}$

$C_{\mathrm{p}}$

$C_{\mathrm{T}}$

$C_{0}$

$D_{\mathrm{L}}$

J

I

$\boldsymbol{J}_{m}$

$\boldsymbol{J}_{q}$

$k$

$K_{\mathrm{T}}$

$N_{\mathrm{A}}$

$N_{\mathrm{i}}$

$N_{\mathrm{L}}$

$N_{\mathrm{T}}$

$R$

$r_{q}$

$t$

$T$

$t_{n}^{0}$

$U_{q}$

$V_{\mathrm{H}}$

$x, y$

$\beta$

$\gamma$

$\delta$

$\delta_{0}$

$\delta_{1}$

$\delta_{F}$

$\Delta g_{b}$

$\Delta W_{B}$ lattice parameter for $\mathrm{BCC} F \mathrm{Fe}(\mathrm{m})$

molar mass of $\mathrm{Fe}(\mathrm{kg} / \mathrm{mol})$

total hydrogen content, expressed as atoms per unit of volume $\left(1 / \mathrm{m}^{3}\right)$

diffusible content of hydrogen $\left(1 / \mathrm{m}^{3}\right)$

specific heat $(\mathrm{J} /(\mathrm{K} \mathrm{kg}))$

trapped content of hydrogen $\left(1 / \mathrm{m}^{3}\right)$

initial content of hydrogen $\left(1 / \mathrm{m}^{3}\right)$

diffusivity of hydrogen in lattice sites $\left(\mathrm{m}^{2} / \mathrm{s}\right)$

Jacobian matrix (m)

identity matrix (1)

hydrogen flux $\left(1 /\left(\mathrm{m}^{2} \mathrm{~s}\right)\right)$

heat flux $\left(\mathrm{W} / \mathrm{m}^{2}\right)$

factor decreasing cohesive strength, based on HEDE mechanism (1)

equilibrium constant (1)

Avogadro constant (1/mol)

shape functions (1)

density of interstitial lattice sites $\left(1 / \mathrm{m}^{3}\right)$

density of trap sites $\left(1 / \mathrm{m}^{3}\right)$

molar gas constant $(\mathrm{J} /(\mathrm{mol} \mathrm{K}))$

heat source $\left(\mathrm{W} / \mathrm{m}^{3}\right)$

time (s)

thermodynamic temperature $(\mathrm{K})$

limit stress of the cohesive element $(\mathrm{Pa})$

internal thermal energy per unit mass $(\mathrm{J} / \mathrm{kg})$

molar volume fraction of hydrogen in $\mathrm{Fe}\left(\mathrm{m}^{3} / \mathrm{mol}\right)$

physical coordinates $(\mathrm{m})$

number of interstitial lattice sites per Fe atom (1)

coefficient for the estimation of the dislocation density as a function of the plastic strain $\left(1 / \mathrm{m}^{3}\right)$

relative displacement between the cohesive element faces (m)

displacement at the end of the elastic behaviour of the cohesive element (m)

displacement at the end of the softening behaviour of the cohesive element (m)

displacement at the final failure of the cohesive element $(\mathrm{m})$

variation of Gibbs free energy $(\mathrm{J} / \mathrm{mol})$

binding enthalpy $(\mathrm{J} / \mathrm{mol})$ 


$\begin{array}{ll}\varepsilon_{p} & \text { plastic strain }(1) \\ \theta & \text { hydrogen coverage factor (1) } \\ \xi, \eta & \text { isoparametric coordinates }(1) \\ \rho & \text { mass density }\left(\mathrm{kg} / \mathrm{m}^{3}\right) \\ \bar{\rho} & \text { dislocation density }\left(1 / \mathrm{m}^{2}\right) \\ \bar{\rho}_{0} & \text { dislocation density for annealed material, i.e. with } \varepsilon_{p}=0\left(1 / \mathrm{m}^{2}\right)\end{array}$

List of acronyms

$\mathrm{C}(\mathrm{T}) \quad$ compact tension specimen

CTOD crack tip opening displacement

HEDE Hydrogen Enhanced DE-cohesion, embrittlement mechanism

HELP Hydrogen Enhanced Localized Plasticity, embrittlement mechanism

MPC multi-point constraint

TSL Traction-Separation Law

\section{Introduction}

Hydrogen embrittlement is a particularly deleterious phenomenon that induces loss of ductility, strength, and fracture toughness [1] when metals are exposed to hydrogen sources. This is an issue quite common in several applications, i.e. transport and storage units where hydrogen is used as energy vector to replace fossil fuels, as well as installations and pipelines in the oil \& gas field.

The hydrogen embrittlement is a complex fully coupled stress-diffusion phenomenon tackled in literature at different dimensional scales with different purposes. In particular, at the macroscale level, Cohesive Zone Models (CZM), initially introduced by [2], are well-accepted numerical techniques to predict the decay of the mechanical properties of a steel in hydrogen-contaminated environment. The main feature of cohesive elements, connected with continuum elements at the region of crack propagation, is the Traction-Separation Law (TSL) that allows for the damage estimation. In particular, damage and failure can be simulated adjusting the TSL cohesive parameters, such as cohesive strength and element critical separations.

Among the cohesive zone models, two main approaches to simulate hydrogen embrittlement have been proposed in literature, $i$ ) a weakly coupled stress-diffusion formulation, with subsequent analyses and ii) a fully coupled formulation that solves the two phenomena in a single-step analysis.

The former approach was first proposed by Olden et al. [3] in 2007, for the estimation of crack initiation in $25 \% \mathrm{Cr}$ duplex stainless steel. Later, the same authors applied this method also to the study of hydrogen embrittlement effect on steel welded joint [4-6].

The latter approach relies on the analogy between thermal-stress analysis and diffusion stressanalysis implemented through customised codes. L. Jemblie et al. [7] proposed an interesting review of coupled diffusion-cohesive zone models, discussing the influence of the main input parameters in hydrogen embrittlement simulation with a sensitivity analysis. Brocks et al. [8], the 
pioneers of this method, investigated the effect of the deformation rate on the hydrogen embrittlement process of a low alloy steel, FeE 690T. This is the only model that implements both the Hydrogen-Enhanced DEcohesion (HEDE) [9] and the Hydrogen Enhanced Localized Plasticity (HELP) [10] mechanisms. Later, Moriconi et al. [11] applied the same model extending the application to cyclic load on a martensitic stainless steel, 15P-5. More recently, Del Busto et al. [12] studied the material degradation and failure due to cyclic load, highlighting the effect of the frequency on a general iron-based material. In addition, Barrera et al. [13] underlined the detrimental effect of hydrogen at the notch of a 2D plane strain plate with HELP mechanism. A combination of analytical and cohesive zone models were adopted by [14, 15] to address the hydrogen assisted stress corrosion crack issue in both CT and circumferentially notched tensile (CNT) specimens. Similarly, D. K. Singh et al. [16, 17] developed an analogous model, both 3D and 2D axisymmetric, to predict the critical stress intensity factors for hydrogen-assisted fracture in HSLA steel (AISI 4340).

In this framework, the current paper, companion of a previous one [18], presents the fully coupled implementation developed by the authors in the Abaqus/Standard finite element software. The additional value, which is also the innovative aspect of the present paper with respect to the aforementioned references, is the fact that the code is released in open source form. The purpose is to ensure the reproducibility of the results as well as to encourage the use of the developed implementation that could also be extended to different applications by the users. To this end, the authors archived all the routines, instructions and examples in [19]. Therefore, similarly to the previous paper, the aim is to provide a guideline to the methodology and to the use of the code, through a practical example. The fully coupled formulation, as the weakly coupled one, was developed within the Polihydra Project to simulate the fracture toughness tests of a C(T) specimen made of AISI 4130 (UNS G41300) steel, charged with atomic hydrogen. Both the models use the experimental data presented in [20] as a benchmark. With this 2D model, the effect of hydrogen is simulated by means of HEDE mechanism. More details concerning this point will be provided in Section 3.2 .

To underline the differences between the fully coupled approach of this paper, it is worth to briefly summarise the weakly coupled approach of [18]. This latter approach consists in three subsequent simulations: $i$ ) a static analysis performed considering the properties of the as-received material to determine the stress field, $i i$ ) a diffusion analysis that assesses the hydrogen concentration based on the stress field previously calculated and iii) a static analysis with cohesive elements to simulate the crack propagation. In this last step, the hydrogen concentration computed in the second simulation is imported as a predefined field and remains constant during crack propagation. Therefore, the model is only partially coupled, because the calculation of hydrogen concentration is not updated to account for the changing state of stress during crack propagation.

The paper is divided into three main parts. An initial part is dedicated to the description of the analogy between thermal and diffusion analyses and the main characteristic equations. This section also discusses the adopted subroutines, their interaction and the main variables for the estimation of the final hydrogen effect, such as the hydrostatic stress gradient.

The second part reports a simple case of a bending strip, used to test the hydrostatic stress gradient on solid elements. Considerations on the numerical errors embedded in the FE formulation are herein provided. 


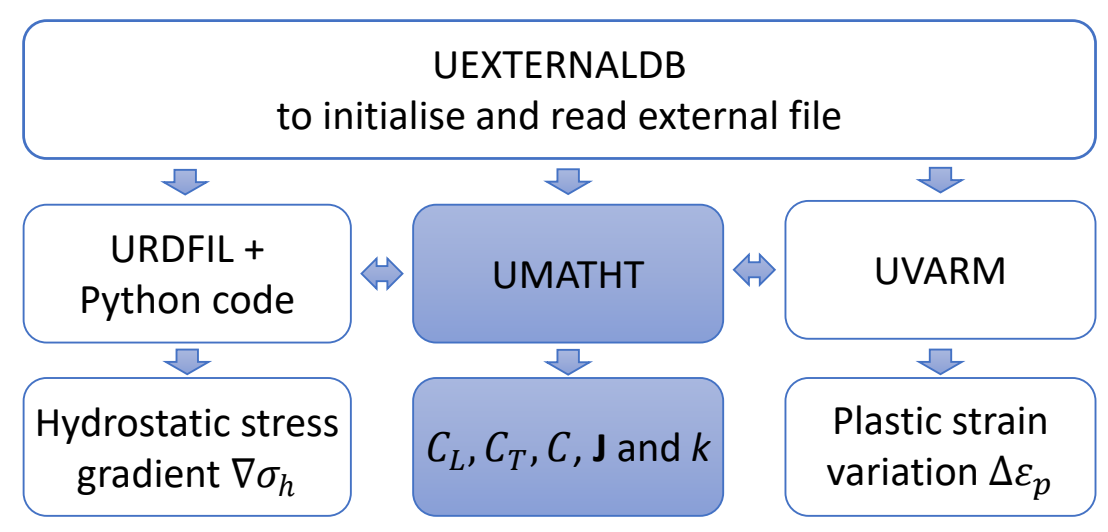

Figure 1: Flowchart of the subroutines used in the code.

Eventually, the third part presents an application of the developed implementation: the fracture toughness model of a $\mathrm{C}(\mathrm{T})$ specimen charged with atomic hydrogen. At the end of this section, a sensitivity analysis is provided to show the capability of the model to estimate the hydrogen embrittling effect as a function of different input parameters.

\section{Model framework}

In Abaqus a fully coupled mass diffusion-stress analysis is currently not implemented. However, due to the analogy between mass diffusion and heat transfer equations, it is possible to exploit the fully coupled thermal-stress analysis available in Abaqus. Nevertheless the governing equations for the hydrogen embrittlement phenomenon are quite specific, so that an extensive use of "user defined" materials and variables has to be implemented by means FORTRAN subroutines.

Figure 1 represents a flowchart that summarises the used subroutines and their primary function. The body of the code consists in the UMATHT subroutine. It allows defining the thermal behaviour of the material and thus, according to the analogy, the equations that couples hydrogen diffusion with stress and strain fields. These fields are calculated by URDFIL and UVARM subroutines and shared with the UMATHT by means of FORTRAN common blocks. The auxiliary UEXTERNALDB subroutine allows for reading external file and initialising some of the variables used during the calculations. In the next sections, each subroutine is presented and discussed separately.

\subsection{Hydrogen diffusion - UMATHT subroutine}

The fully coupled implementation relies on the analogy between heat transfer and mass diffusion equations. The hydrogen content continuity equation can be written as

$$
\frac{\partial C}{\partial t}+\nabla \cdot J_{m}=0
$$


where $C$ is the hydrogen content expressed as number of atoms per unit volume and $\boldsymbol{J}_{m}$ the hydrogen flux. For the current implementation there is no hydrogen source term, since the code was developed to simulate hydrogen precharged specimens. Similarly, the continuity equation for heat transfer implemented in Abaqus reads

$$
\rho \frac{\partial U_{q}}{\partial t}+\nabla \cdot J_{q}+r_{q}=0
$$

with $\rho$ mass density, $U_{q}$ internal thermal energy per unit of mass, $\boldsymbol{J}_{q}$ heat flux, and $r_{q}$ heat sources. Therefore, a basic first set of analogies can be established:

$$
\begin{aligned}
\rho U_{q} & \equiv C \\
\boldsymbol{J}_{q} & \equiv \boldsymbol{J}_{m}
\end{aligned}
$$

with $r_{q}=0$, and, for the sake of simplicity, $\rho=1$.

It is now necessary to find an expression for the mass flux $\boldsymbol{J}_{m}$ to be implemented in Abaqus. As already mentioned in the previous paper [18], the hydrogen concentration includes two contributions: the diffusible hydrogen, $C_{\mathrm{L}}$, and the trapped hydrogen, $C_{\mathrm{T}}$ :

$$
C=C_{\mathrm{L}}+C_{\mathrm{T}}
$$

The interstitial diffusion of hydrogen is driven by both the concentration and hydrostatic stress gradients [21]. Therefore, the hydrogen flux vector can be written as

$$
J_{m}=-D_{\mathrm{L}} \nabla C_{\mathrm{L}}+\frac{D_{\mathrm{L}} C_{\mathrm{L}} V_{\mathrm{H}}}{R T} \nabla \sigma_{H}
$$

where $D_{\mathrm{L}}$ is the effective diffusivity of interstitial concentration, $V_{\mathrm{H}}$ is the partial molar volume, $R$ is the universal gas constant, $T$ is the thermodynamic temperature and $\sigma_{\mathrm{H}}$ is the hydrostatic stress.

According to the Oriani's theory [22], the trapped hydrogen concentration, $C_{\mathrm{T}}$, can be written as a function of the diffusible concentration, $C_{\mathrm{L}}$ as:

$$
C_{\mathrm{T}}=\frac{N_{\mathrm{T}} K_{\mathrm{T}} C_{\mathrm{L}}}{\left(K_{T}-1\right) C_{\mathrm{L}}+N_{\mathrm{L}}}
$$

where $N_{\mathrm{L}}$ is the density of interstitial lattice sites, $N_{\mathrm{T}}$ is the trap site density and $K_{T}$ is the equilibrium constant, evaluated as a function of the binding enthalpy $\Delta W_{B}$ [22]:

$$
K_{\mathrm{T}}=\exp \frac{-\Delta W_{\mathrm{B}}}{R T}
$$

$\Delta W_{B}$ is set equal to $-60 \mathrm{~kJ} / / \mathrm{mol}$ [23].

Moreover, for $\alpha-\mathrm{Fe}$, according to [24], $N_{\mathrm{L}}$ is:

$$
N_{\mathrm{L}}=\frac{N_{\mathrm{A}} \beta \rho}{A_{r}}
$$


where $N_{\mathrm{A}}$ is the Avogadro constant, $\beta$ is the number of interstitial lattice sites per Fe atom (6, under the hypothesis of occupation of the tetrahedral sites by hydrogen atoms at room temperature), $\rho$ is the iron density $\left(7.87 \times 10^{3} \mathrm{~kg} / \mathrm{m}^{3}\right.$ at $293 \mathrm{~K}$ [24] $)$ and $A_{r}$ is the molar mass of $\mathrm{Fe}$ $\left(55.8 \times 10^{-3} \mathrm{~kg} / \mathrm{mol}\right.$ at $\left.293 \mathrm{~K}\right)[11]$.

Two methods were used for the estimation of the number of trap sites $N_{\mathrm{T}}$. The first one is based on the experimental work by Kumnick and Johnson [23]. From this, [21] and [24] proposed the following exponential law, evaluating the density of dislocation trap sites from the equivalent plastic strain, $\varepsilon_{p}$ :

$$
\log _{10} N_{\mathrm{T}}=23.26-2.33 \cdot \exp \left(-5.5 \cdot \varepsilon_{p}\right)
$$

The second method is the one proposed by Sofronis et al. [25]:

$$
N_{\mathrm{T}}=\sqrt{2} \cdot \frac{\bar{\rho}}{\bar{a}}
$$

where $\bar{a}=2.86 \times 10^{-10} \mathrm{~m}$ is the lattice parameter of BCC iron and $\bar{\rho}$ is the dislocation density. In turn $\bar{\rho}$ is a function of the equivalent plastic strain:

$$
\bar{\rho}= \begin{cases}\bar{\rho}_{0}+\gamma \varepsilon_{p} & \text { for } \varepsilon_{p}<0.5 \\ \bar{\rho}_{1} & \text { for } \varepsilon_{p} \geq 0.5\end{cases}
$$

where $\bar{\rho}_{0}=1 \times 10^{10} \mathrm{~m} / \mathrm{m}^{3}$ is the dislocation density for annealed material with $\varepsilon_{p}=0, \bar{\rho}_{i}=$ $1 \times 10^{16} \mathrm{~m} / \mathrm{m}^{3}$ is the maximum dislocation density, and $\gamma=2 \times 10^{16} \mathrm{~m} / \mathrm{m}^{3}$.

By analysing eq. (5) it can be observed that the forces driving the mass flux $\boldsymbol{J}_{m}$ are $\nabla C_{\mathrm{L}}$ and $\nabla \sigma_{\mathrm{H}}$. While the latter can be obtained from the coupled stress analysis, $\nabla C_{\mathrm{L}}$ has to be solved along (1): it is therefore natural to assume $C_{\mathrm{L}}$ as the primary unknown, and to compute $C_{\mathrm{T}}$ from (6). Consequently, the mass diffusion-thermal analogy, first introduced in (3) can be completed by this last statement:

$$
T \equiv C_{\mathrm{L}}
$$

Further analogies are not possible. In fact, in Abaqus the basic material thermal model refers to the Fourier law, which is inadequate for implementing the complex behaviour described by eqs. (5)-(11). Consequently, an advanced thermal material model has to be implemented by user subroutine UMATHT.

Abaqus user material subroutines are called at each attempted time increment $t+\Delta t$, (converged or not,) and at each element integration point. The most important variables of UMATHT are summarised in table 1, along with their interpretation in standard thermal and mass diffusion analyses. These variables can be divided in two groups.

- input variables ( intent (in) in Fortran90), passed in as information: TEMP, DTEMP, DTEMDX, TIME, DTIME.

- input-output variables (intent (inout)) to be updated by the user subroutine: U, DUDT, DUDG, FLUX, DFDT, DFDG. These variables contain on input the values of the corresponding quantities at time $t$ and have to be updated by the user subroutine with the value at time $t+\Delta t$. 
Table 1: Analogy between thermal and mass diffusion variables in UMATHT user subroutine. The fist part of the table presents the input-output variables; the second part the input variables.

\section{Variable Heat transfer Mass diffusion}

\begin{tabular}{lccl} 
U & $U_{q}$ & $C_{\mathrm{L}}+C_{\mathrm{T}}$ & \\
DUDT & $\frac{\partial U_{q}}{\partial T}$ & $\frac{\partial\left(C_{\mathrm{L}}+C_{\mathrm{T}}\right)}{\partial C_{\mathrm{L}}}$ & $=1+\frac{N_{\mathrm{T}} N_{\mathrm{L}} K_{T}}{\left(N_{\mathrm{L}}+C_{\mathrm{L}}\left(K_{T}-1\right)\right)^{2}}$ \\
DUDG & $\frac{\partial U_{q}}{\partial \nabla T}$ & 0 & \\
FLUX & $\boldsymbol{J}_{q}(T, \nabla T)$ & $\boldsymbol{J}_{m}\left(C_{\mathrm{L}}, \nabla C_{\mathrm{L}}\right)$ & $=-D_{\mathrm{L}} \nabla C_{\mathrm{L}}+\frac{D_{\mathrm{L}} C_{\mathrm{L}} V_{\mathrm{H}}}{R T} \nabla \sigma_{\mathrm{H}}$ \\
DFDT & $\frac{\partial \boldsymbol{J}_{q}}{\partial T}$ & $\frac{\partial \boldsymbol{J}_{m}}{\partial C_{\mathrm{L}}}$ & $=\frac{D_{\mathrm{L}} V_{\mathrm{H}}}{R T} \nabla \sigma_{\mathrm{H}}$ \\
DFDG & $\frac{\partial \boldsymbol{J}_{q}}{\partial \nabla T}$ & $\frac{\partial \boldsymbol{J}_{m}}{\partial \nabla C_{L}}$ & $=-D_{L} \boldsymbol{I}$ \\
\hline TEMP & $T$ & $C_{\mathrm{L}}$ & \\
DTEMP & $\Delta T$ & $\Delta C_{\mathrm{L}}$ & \\
DTEMDX & $\nabla T$ & $\nabla C_{\mathrm{L}}$ & \\
TIME & $t$ & $t$ \\
DTIME & $\Delta t$ & $\Delta t$ &
\end{tabular}


For the present problem it holds

$$
C(t+\Delta t)=C(t)+\frac{\partial C}{\partial C_{L}} \Delta C_{L}+\frac{\partial C}{\partial N_{T}} \frac{d N_{T}}{d \varepsilon_{p}} \Delta \varepsilon_{p}
$$

where:

$$
\begin{aligned}
\frac{\partial C}{\partial C_{L}} & =1+\frac{N_{T} K_{T} N_{L}}{\left(K_{T} C_{L}+N_{L}\right)^{2}} \\
\frac{\partial C}{\partial N_{T}} & =\frac{K_{T} C_{L}}{K_{T} C_{L}+N_{L}}
\end{aligned}
$$

As for $\frac{d N_{T}}{d \varepsilon_{p}}$, one has

$$
\frac{d N_{T}}{d \varepsilon_{p}}=2.95 \cdot 10^{-8} \exp \left(-5.5 \varepsilon_{p}\right) \cdot 10^{23.26-2.33 \exp \left(-5.5 \varepsilon_{p}\right)}
$$

or

$$
\frac{d N_{T}}{d \varepsilon_{p}}= \begin{cases}\sqrt{2} \frac{\gamma}{a} & \text { for } \varepsilon_{p}<0.5 \\ 0 & \text { for } \varepsilon_{p} \geq 0.5\end{cases}
$$

depending on the method adopted to estimate $N_{T}$. The variation of the equivalent plastic strain, $\Delta \varepsilon_{p}$, is calculated during the analysis by the user subroutine UVARM as a difference between the current plastic strain value and the one of the previous time increment.

The UMATHT subroutine, besides the expression of the hydrogen flux, $\boldsymbol{J}_{m}$, requires the definition of its derivative with respects to both the concentration and the concentration gradient:

$$
\begin{aligned}
\frac{\partial \boldsymbol{J}_{m}}{\partial C_{\mathrm{L}}} & =\frac{D_{\mathrm{L}} V_{H}}{R T} \nabla \sigma_{\mathrm{H}} \\
\frac{\partial \boldsymbol{J}_{m}}{\partial \nabla C_{\mathrm{L}}} & =-D_{\mathrm{L}} \boldsymbol{I}
\end{aligned}
$$

with $I$ the identity tensor. The gradient of the hydrostatic stress is calculated separately with the user subroutine URDFIL and imported in the UMATHT subroutine through a FORTRAN common block. In section 2.3, details related to the calculation of the hydrostatic stress field in Abaqus software will be discussed.

The effect of hydrogen in the cohesive elements is taken into account by means of the following relationships [26], as done also for the weakly coupled formulation [18]. In particular, the total hydrogen concentration, $C$, is related to the surface concentration by:

$$
\theta=\frac{C}{C+\exp \left(-\Delta g_{b} / R T\right)}
$$

where $\Delta g_{\mathrm{b}}$ is the variation of Gibbs free energy, set equal to $30 \mathrm{~kJ} / \mathrm{mol}$ [26]. The hydrogen coverage factor $\theta$, is then used to resize the TSL as:

$$
k=\frac{t_{n}^{0}(\theta)}{t_{n}^{0}(0)}=1-1.0467 \cdot \theta+0.1687 \cdot \theta^{2}
$$




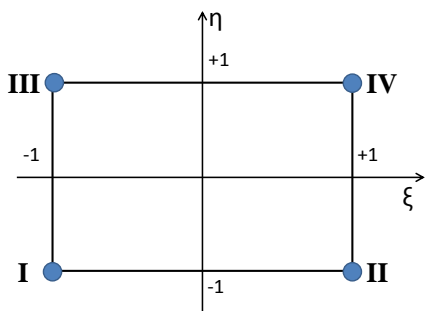

Figure 2: Scheme of a $2 D$ reference quadrilateral element in isoparametric coordinates with 4 integration points indicated with Roman numbers; the origin of the coordinates system coincides with the unique integration point in the reduced integration formulation.

\subsection{Increment of equivalent plastic strain, UVARM subroutine}

The value of the plastic strain $\varepsilon_{p}$ in Eqs. (9) and (11), is interpreted as the "equivalent plastic strain" that Abaqus saves in variable PEEQ, i. e. a monotonically increasing scalar variable that is the work-conjugate of the yield equivalent stress and is responsible of plastic dissipation. In eq. (13), the increment $\Delta \varepsilon_{p}$ is required: this is calculated with the user subroutine UVARM that allows defining general output quantities as a function of available integration point output variables. Subroutine UVARM is called at the end of a converged time increment $t+\Delta t$. The values of PEEQ at time $t$ and $t+\Delta t$ are saved in a FORTRAN common block which is accessed by the UMATHT user subroutine where the difference $\Delta \varepsilon_{p}=\Delta \mathrm{PEEQ}$ is computed and substituted in eq. (13). It should be noted here that subroutine UMATHT is called when the time increment is not yet converged, so that actually the present implementation uses $\Delta \varepsilon_{p}$ estimated at the preceding converged increment. However, for small $\Delta t$ the associated error is considered negligible.

\subsection{Calculation of hydrostatic stress gradient, URDFIL subroutine}

It is well known that an accurate reconstruction of the stress field and its gradient is not straightforward for finite element approximations. In the present code a simple but effective approximation is adopted:

- first the stress field is extrapolated at nodes and averaged, and then

- it is interpolated on the finite element using the displacement shape functions. Stress field derivatives are computed simply by derivation of the shape functions.

The nodal averaged stress values of the hydrostatic stress are stored on disk by Abaqus at the end of a converged time increment; these values are then read by user subroutine URDFIL, in order to compute the spatial stress gradient at the integration points. The computed values are stored in a common block and made available to the UMATHT subroutine. 
Considering $N_{\mathrm{i}}(\xi, \eta)$ the shape functions of a $2 D$ reference quadrilateral element in isoparametric coordinates $(\xi, \eta)$ as in Figure 2, their expressions are:

$$
\begin{aligned}
N_{I}(\xi, \eta) & =\frac{1}{4}(1-\xi)(1-\eta) \\
N_{I I}(\xi, \eta) & =\frac{1}{4}(1+\xi)(1-\eta) \\
N_{I I I}(\xi, \eta) & =\frac{1}{4}(1-\xi)(1+\eta) \\
N_{I V}(\xi, \eta) & =\frac{1}{4}(1+\xi)(1+\eta)
\end{aligned}
$$

The nodal values of the extrapolated field are then averaged based on the connectivity matrix among adjacent elements. Abaqus automatically evaluates the field averaged at nodes that can be stored during the analysis in a .fil file. Concerning the current implementation, the hydrostatic stress field averaged at the nodes is saved in a fil file. The user subroutine URDFIL accesses the .fil file and calculates the hydrostatic stress gradient by using the partial derivatives of the shape functions, as follows:

$$
\begin{aligned}
& \frac{\partial \sigma_{H}(\xi, \eta)}{\partial \xi}=\sum_{i=1}^{4} \frac{\partial N_{i}(\xi, \eta) \sigma_{H, i}}{\partial \xi}=\sum_{i=1}^{4} N_{i, \xi}(\xi, \eta) \sigma_{H, i} \\
& \frac{\partial \sigma_{H}(\xi, \eta)}{\partial \eta}=\sum_{i=1}^{4} N_{i, \eta}(\xi, \eta) \sigma_{H, i}
\end{aligned}
$$

These expressions, valid in each point of the element, for the developed implementation are evaluated at the unique integration point centered in $\xi=\eta=0$. Finally, the following formulation is used to calculate the gradient of the hydrostatic stress in the global coordinates system $(x, y)$ :

$$
\nabla \sigma_{H}(x, y)=\mathbf{J}^{-1} \cdot \nabla \sigma_{H}(\xi, \eta)
$$

where $\mathbf{J}$ is the Jacobian matrix that includes the partial derivatives of the global coordinates $(x, y)$ with respect to the isoparametric coordinates $(\xi, \eta)$ :

$$
\begin{aligned}
& \frac{\partial x}{\partial \xi}=\sum_{i=1}^{4} \frac{\partial\left[N_{i}(\xi, \eta) x_{i}\right]}{\partial \xi}=\sum_{i=1}^{4} N_{i, \xi}(\xi, \eta) x_{i} \\
& \frac{\partial x}{\partial \eta}=\sum_{i=1}^{4} N_{i, \eta}(\xi, \eta) x_{i} \\
& \frac{\partial y}{\partial \xi}=\sum_{i=1}^{4} N_{i, \xi}(\xi, \eta) y_{i} \\
& \frac{\partial y}{\partial \eta}=\sum_{i=1}^{4} N_{i, \eta}(\xi, \eta) y_{i}
\end{aligned}
$$


The calculations of the partial derivatives of the shape functions and the $\mathbf{J}^{-\mathbf{1}}$ are carried out by a Python script, run before the Abaqus analysis. This approach is valid assuming small displacements. On the contrary, the $\mathbf{J}^{-1}$ calculation should be embedded into the code in order to determine it increment by increment during the analysis. The main advantage of this technique is the possibility to adopt not only the full but also the reduced integration for element formulation, decreasing the computational time.

\section{Examples}

This section is dedicated to the presentation and the discussion of two examples. The first one explains the importance of the mesh regularity for a proper estimation of the hydrostatic stress gradient. The second one shows a possible application for the developed fully coupled implementation: a model simulating a fracture toughness test of $\mathrm{C}(\mathrm{T})$ specimen charged with $1.5 \mathrm{ppm}$ of atomic hydrogen. A sensitivity analysis of the model to the main input parameters is then performed to explore the capability of the model as predictive tool.

The codes for running these simulations have been archieved in [19] along with detailed stepby-step instructions. Table A.2 in Appendix A summarizes all the relevant input parameters.

\subsection{Example 1-Considerations on mesh refinement}

The quantification of the diffusible hydrogen concentration, $C_{L}$, relies on the hydrostatic stress gradient calculation. From a computation point of view, the hydrostatic stress field is evaluated at the integration points of solid 2D plane strain elements, and then transferred to the cohesive elements via subroutines. This example underlines the importance of the mesh regularity to correctly compute the hydrostatic stress gradient, thereby ensuring a proper estimation of $C_{L}$, as well.

The example consists in a simple square plate subjected to a bending moment, divided in two regions with different mesh refinement (Figure 3). Only a quarter (unit size, $1 \mathrm{~mm} \times 1 \mathrm{~mm}$ ) of the plate is modelled, applying anti-symmetric and symmetric boundary conditions at the nodes along $x$ and $y$ axes, respectively. A distributed triangular load linearly variable from 0 to 1 with $y$ coordinate is applied to the right side of the $2 \mathrm{D}$ plate. Two regions, $A$ and $B$, with different mesh size are considered. In particular, region $A$ has element size equal to $0.05 \mathrm{~mm}$, while region $B$ has $0.1 \mathrm{~mm}$ element size. Simulations with coarser or more refined mesh sizes were also performed, leading to considerations similar to those herein presented.

Figure 4 shows a uniform mesh together with three different mesh refinement techniques, implemented in the example. These are the most commonly used methods:

1. with diagonal partition,

2. with interposition of trapezoidal elements and

3. with linear Multi-Point Constraints (MPC).

The MPC considers identical sizes for elements within the same region. Passing from B to A region, the refinement is abrupt to half the size, and nodes at the boundaries between the regions are connected with linear MPCs. This technique allows for a structured mesh (within each refinement zone) with equal-sided elements, so that no mesh distortion is introduced. 


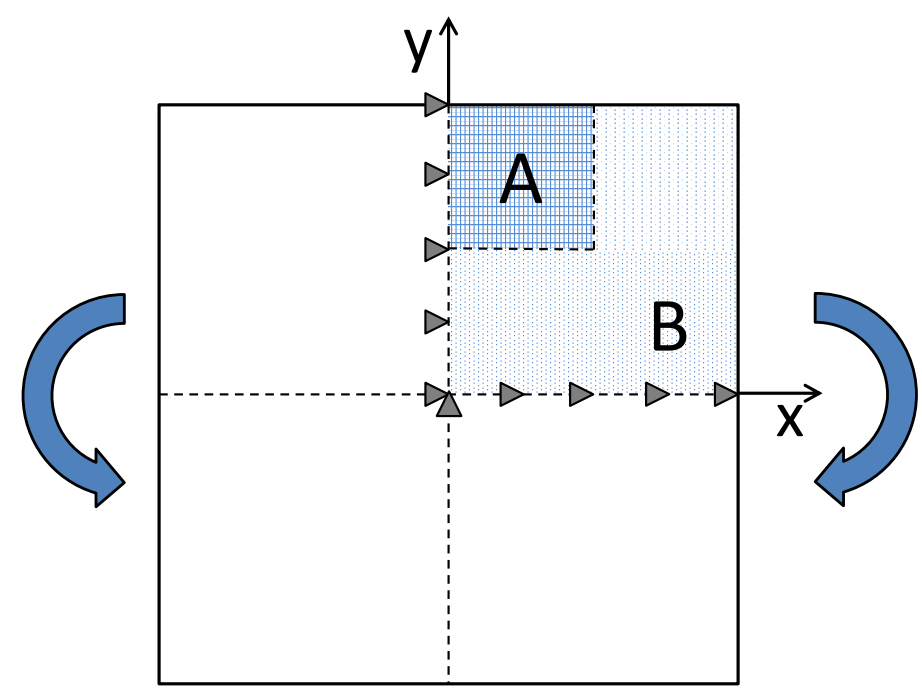

Figure 3: Geometry of the bending example. The model consists in one quarter of the plate; two regions are considered: $A$ has half sized elements with respect to $B$.

The quality of the three different meshes are evaluated considering the percentage error in the estimation of the hydrostatic stress gradient along $y$ axis, with respect to the analytical solution. The contours in Figure 4 highlight, for all the cases, the most critical regions, listed and commented below.

1. Elements along the upper and lower edges for all the mesh types. In these regions, it is well known that finite elements provide less accurate solution, due to the node averaging of the computed field [27]. Indeed, this is valid also for the uniform mesh. The edge elements underestimate the stress gradient between $-50 \%$ and $-62 \%$.

2. Distorted elements, as the diagonal elements in Mesh 1 (Figure $4 \mathrm{~b}$ ) and the trapezoidal elements in Mesh 2 (Figure 4c). Here, the stress gradient alternates over- and under-estimations of the solution of $\pm 20 \%$.

3. Horizontal elements on the edge in Mesh 3 (Figure 4 $\mathrm{d}$ ). In correspondence of these elements, where the MPC is active, the stress solution is less precise and underestimated by $-13 \%$.

In conclusion, Mesh 3 with MPC entails the lowest error in the evaluation of the hydrostatic stress gradient and therefore is the most suitable and advisable mesh refining technique to be used.

\subsection{Example 2 - Application and validation of the model}

The second example consists in a model, which simulates the fracture toughness test of a $\mathrm{C}(\mathrm{T})$ specimen charged with $1.5 \mathrm{ppm}$ of atomic hydrogen. This example intends to reproduce the experimental results presented in [20]. Indeed, the data of tests performed both in as-received 


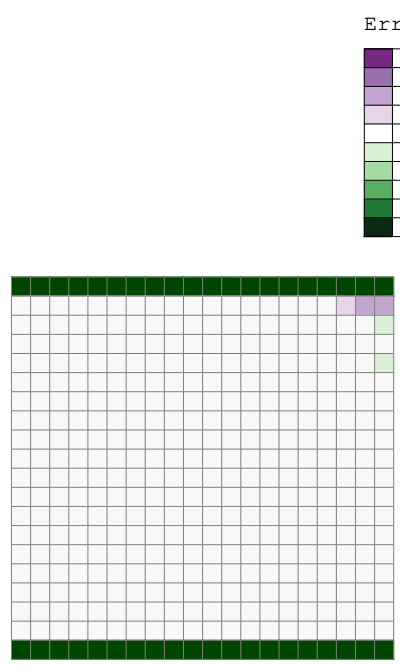

(a)

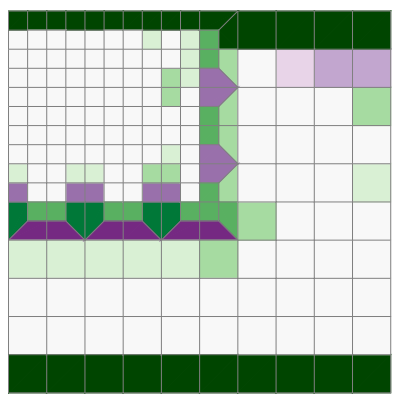

(c)

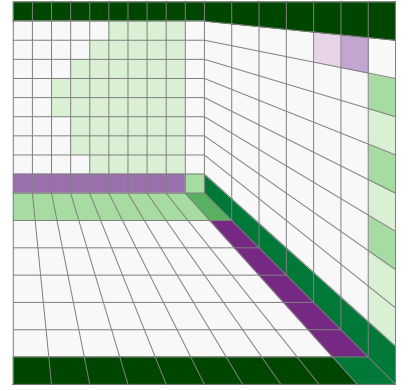

(b)

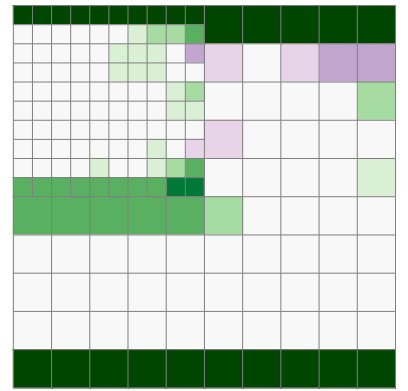

(d)

Figure 4: Percentage error in the estimation of the hydrostatic stress gradient $y$ component, $\partial \sigma_{\mathrm{H}} / \partial y$, for four mesh refinements: a) Uniform mesh refinement; b) Mesh 1, with diagonal partition; c) Mesh 2, with trapezoidal elements at the transition; d) Mesh 3, with MPC at the transition. 
and hydrogen pre-charged specimens are used as a benchmark for the numerical model. The example herein presented as an application of the fully coupled implementation was proposed also for the weakly coupled formulation [18]. The aim of this section is, in fact, not only providing a demonstration of the use of the fully coupled code but also comparing the two implementations.

In particular, the example consists in a $2 \mathrm{D}$ cohesive zone model of a $\mathrm{C}(\mathrm{T})$ specimen made of a high strength steel, AISI 4130. The cohesive constitutive law (TSL) that represents phenomenologically the mechanical response of the as-received material in a fracture toughness test is set as input in the model. Based on that, the model predicts the behaviour of the hydrogen contaminated steel. The material property decay, experienced by the embrittled steel, is simulated scaling the TSL according to the calculated total hydrogen concentration. Figure 5 shows the reference and scaled TSL as a function of the coverage factor $\theta$, Eq. (20). This formulation reproduces at a macroscopic dimension level the microstructural HEDE (Hydrogen Enhanced DE-cohesion) mechanism. The fully coupled implementation, herein considered, enables to couple hydrogen diffusion with stress and strain fields in a unique analysis, as explained in Section 2.

The model is symmetric (Figure 6). The solid mesh is connected with cohesive elements along the symmetry plane. The mesh adopts linear quadrilateral elements with plane strain formulation for thermo-mechanical problems (Abaqus identification: CPE4RT). From the edges of the specimen approaching the symmetry plane, the mesh is progressively refined, up to $15.6 \mu \mathrm{m}$ (size of the smallest square elements at the tip), using the MPC technique. This mesh was already used for a weakly coupled implementation [18]. In that case, the concentration of the diffusible hydrogen, $C_{L}$, was estimated based on the hydrostatic stress. Since the fully coupled formulation considers its gradient, this type of mesh is even more precious, in accordance with the discussion in Section 3.1

Regarding cohesive elements, the proposed mesh considers a highly-refined mesh near to the crack tip, with a rectangular shape $(0.53 \mathrm{~mm}$ high and $1.53 \mathrm{~mm}$ long, ahead of the crack tip,). Knowing the experimental crack length at the end of the test, i.e. when the specimen is considered failed and the experimental test interrupted, we have selected specifically this size for the highly-refined region, because the progression of numerical crack simulation loses its meaning. Indeed, according with the simulations run, we can point out the importance of a uniform mesh refinement in the direction of crack propagation (i.e. uniform cohesive element size), in order to avoid numerical instability during the computation of cohesive element failure.

The software Abaqus allows for a tabular definition of the TSL, therefore with an a priori unlimited number of independent parameters. Specifically, we selected the trapezoidal shape, as in [8] and [28]. It is defined by 4 independent parameters, e.g. $t_{n}^{0}, \delta_{0}, \delta_{1}$, and $\delta_{F}$, see Figure 5. With this choice the initial slope and the cohesive energy, i.e. the area below the TSL curve, become dependent parameters.

In the numerical literature, some authors reduce the number of independent parameters for TSL definition, e.g. with fixed ratios between the separations $\delta_{i}$. With reference to the trapezoidal TSL shape, Schwalbe et al. [29] leave only two independent parameters, which can be determined experimentally, e.g. the cohesive energy and the cohesive stress. For the present and the previous [18] papers, the ratios between separations were relaxed, and the TSL parameters were identified as a result of a calibration process that compared the numerical estimations of CTOD- $\Delta a$ (crack tip opening displacement vs crack advancement) with the experimental measurements of 


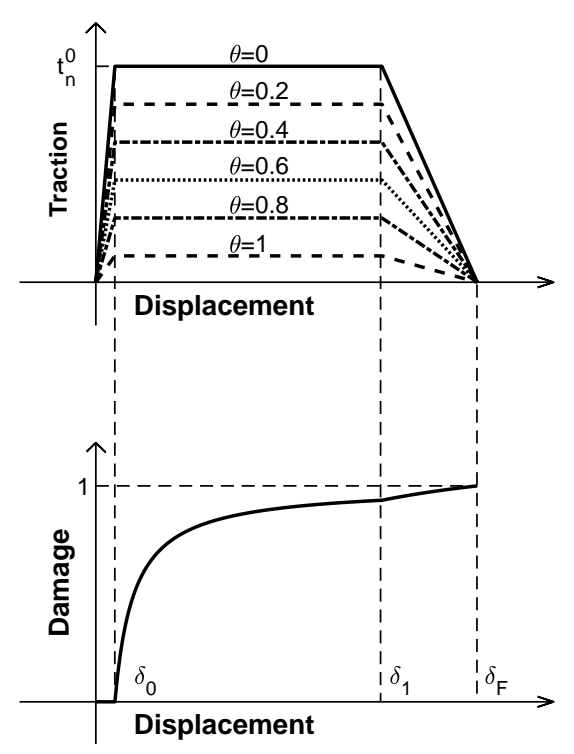

Figure 5: TSL curves as a function of the coverage factor $\theta$, eq. 20, and corresponding Damage curve.

the as-received material [20]. Details of this calibration procedure are given in [18]. The crack is numerically advanced of one-element length when the underlying cohesive element experiences a complete separation, i. e. its vertical displacement is equal to $\delta_{F}$ and the damage is equal to 1 .

The model considers an initial hydrogen concentration $C_{0}$ of $1.5 \mathrm{ppm}$ that is assumed uniform within the specimen, at the beginning of the analysis, and constant during the simulation, at the free edges (Figure 6).

The numerical results are validated comparing the prediction of the CTOD- $\Delta a$ curve with the experimental data of a test carried out on a hydrogen charged specimen [20]. More details are presented in the previous paper [18].

Figure 7 reports the outcome of the fully coupled implementation. For the sake of completeness, the result of the weakly coupled formulation is added, as well. For the specific case, it seems that the numerical prediction is not sensitive to the choice of the formulation, i. e. weakly or fully coupled. Indeed, both the numerical implementations provide similar results. This is reasonable, considering that the main difference between the two formulations is the calculation of $C_{T}$, which is, in this case, considerably lower with respect to $C_{L}$ (as will be discussed later in the text).

It is important to point out that hydrogen deeply influences crack initiation, while the propagation along the symmetry plane of the specimen is extremely sudden. However, from Figure 7 it is evident that both numerical approaches overall underestimate the effect of hydrogen. This could be an intrinsic limitation of the model.

It is well known that the hydrogen embrittlement is a complex microstructure-dependent phenomenon, explained in literature by several possible mechanisms. H-enhanced decohesion (HEDE) [9], [30] states that $\mathrm{H}$ atoms close to the crack tip lower the fracture energy, causing cleavage-like failure. H-enhanced local plasticity (HELP) [31] , [10], [32] is based on the observations of dislocation pile-ups and high local plastic-like deformation in hydrogen embrittled materials. Hydride 


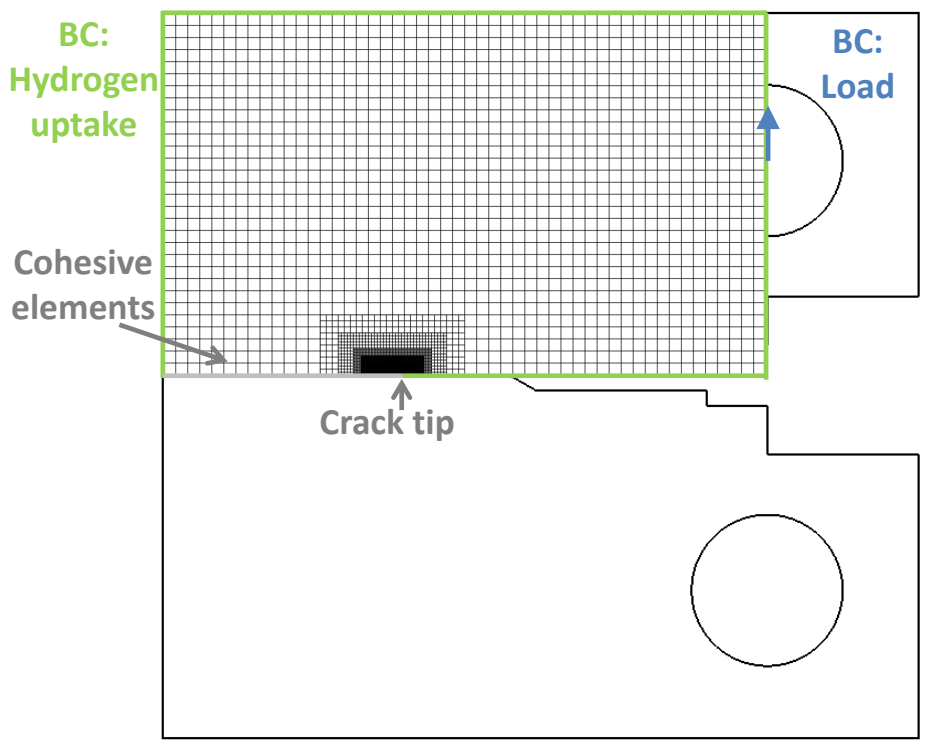

Figure 6: $\mathrm{C}(\mathrm{T})$ geometry and simplification of the model, with boundary conditions.

formation systems are described in [33]. More recently atomistic simulations have been used to propose nanoscale mechanisms for hydrogen embrittlement [34], [35]. A new model, in which the accumulation of hydrogen atoms at the crack tip annuls the ability of the material to blunt cracks, has been proposed in [36].

All the previous mechanisms show contradictory results and in general physically based mechanisms able to quantitatively predict the hydrogen embrittlement do not exist. Besides, not all these mechanisms can be easily implemented in macro-scale FE models. Therefore, it is possible that the HEDE mechanism implemented in the current model is not the only that occurs, since a combination of more mechanisms acting together can also be possible.

An important result that highlights the different features of the two formulations is the contour of the diffusible hydrogen concentration. Figure 8 shows the $C_{L}$ field, calculated with the two approaches, i. e. weakly and fully coupled. The concentration fields are selected at the same instant, the beginning of crack propagation, before the failure of the first cohesive element, when the hydrogen concentration reaches its maximum value. The fully coupled formulation accounts for the mutual influence of the stress-strain state and hydrogen diffusion, increment by increment, during the crack propagation. Therefore, the diffusible hydrogen content is calculated at each position of the crack tip and changes during the analysis. On the other hand, in the weakly coupled implementation, the $C_{L}$ content is computed considering the hydrostatic stress field calculated $a$ priori, before the crack propagation. $C_{L}$ is frozen as constant field, while the crack propagates. In other words, the concentration field of Figure $8 \mathrm{a}$ is invariant during the simulation, while the one of Figure $8 \mathrm{~b}$ is function of the time increment. The two concentration fields are different: the weakly coupled formulation of Figure 8 a depends on the hydrostatic stress; the fully coupled 


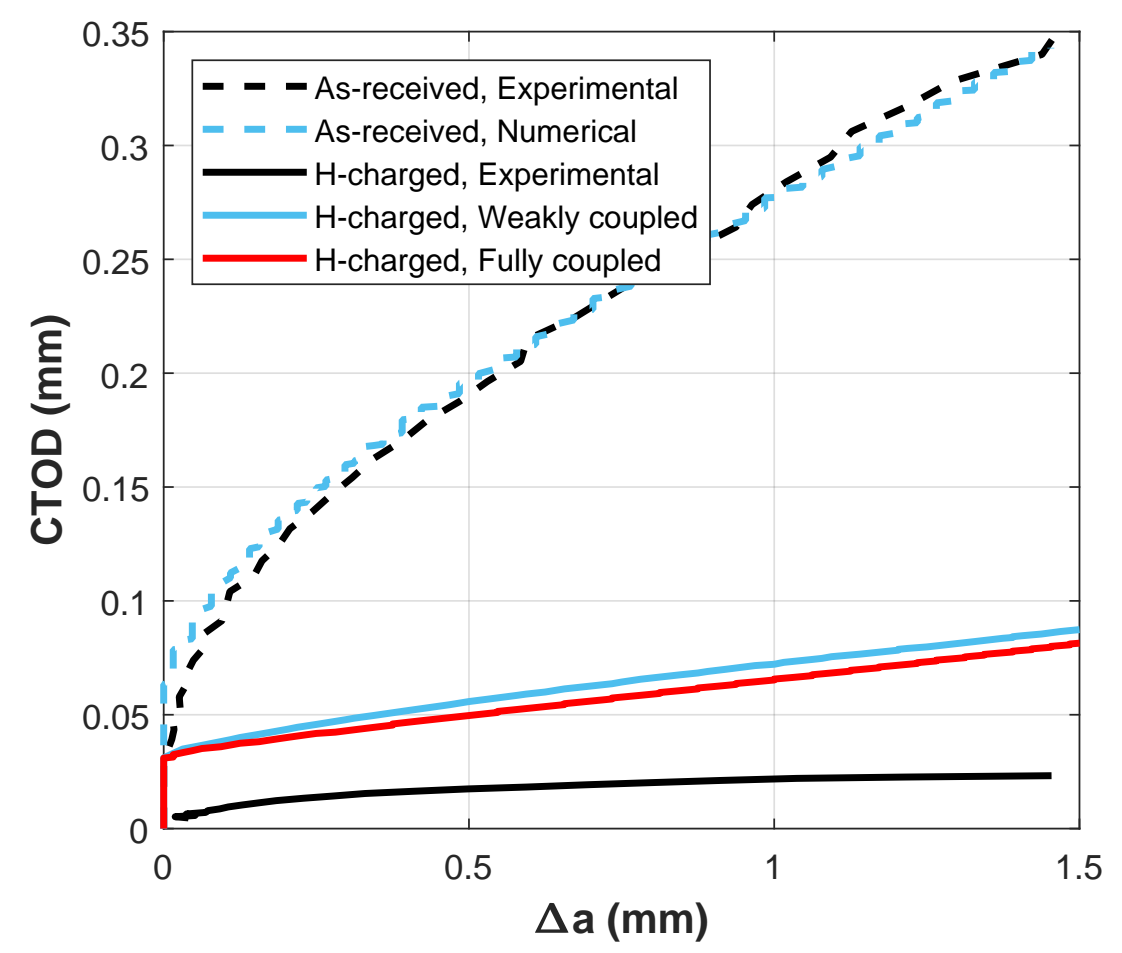

Figure 7: Experimental data, processed from the toughness tests of Ref. [20], and results of numerical simulations in presence of hydrogen $(1.5 \mathrm{ppm})$. Crack tip opening displacement (CTOD) as a function of crack advancement $\Delta a$, numerically estimated with the weakly and the fully coupled formulations. 


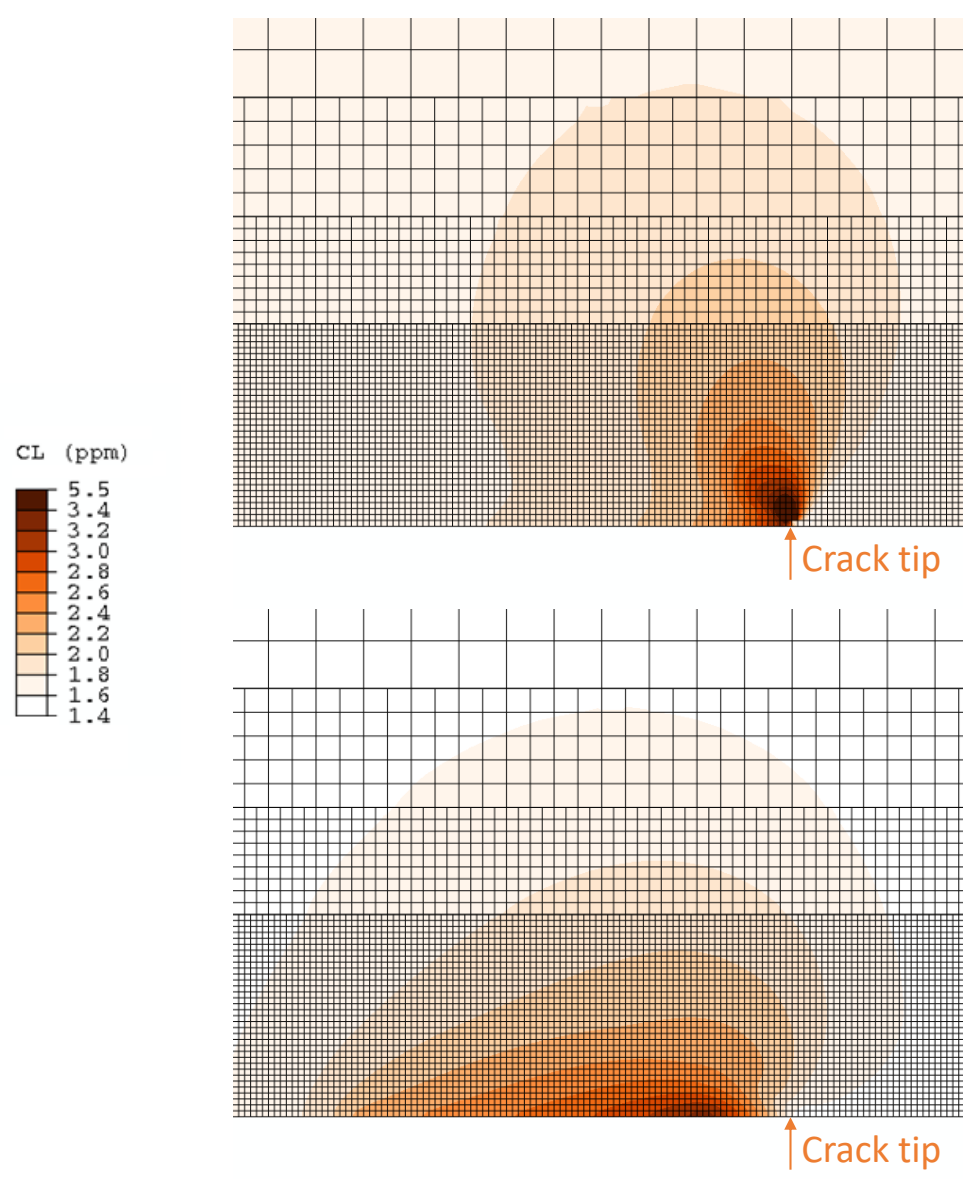

a)

b)

Figure 8: Contour of interstitial hydrogen concentration at the beginning of crack propagation, $C_{L}$ for the a) weakly and b) fully coupled formulations. Magnification at the crack tip.

formulation of Figure $8 \mathrm{~b}$ depends on its gradient. The concentration field of the fully coupled formulation is in agreement with the profile shown in [37]. It is clear that this trend can be estimated only by numerical models, since experimental techniques for point measurements of hydrogen concentration do not exist.

In correspondence of the same time increment, before crack initiation, it is interesting to plot the diffusible hydrogen content, $C_{L}$ and trap site amount, $C_{T}$. Figures 9 (a), (b) show the trend of both concentrations as a function of the distance from the crack tip, for the weakly and the fully coupled formulations, respectively. For the latter, the two methods adopted to compute $C_{T}$, i. e. the Kumnick [23] and the Sofronis [25] ones, are presented. Despite the prediction of the mechanical response in terms of CTOD- $\Delta a$ is similar for the weakly and fully coupled formulations, the estimation of hydrogen concentrations at the crack tips are quite different. In particular:

1. The $C_{T}$ computed with the weakly coupled formulation (Figure $9 \mathrm{a}$ ) is one order of magnitude lower than $C_{L}$. Thus, $C$ (the sum of both) is slightly higher than $C_{L}$, with a peak of $3.3 \mathrm{ppm}$ located at the end of the first element, $15.6 \mu \mathrm{m}$, ahead of the crack tip. 
Weakly coupled formulation

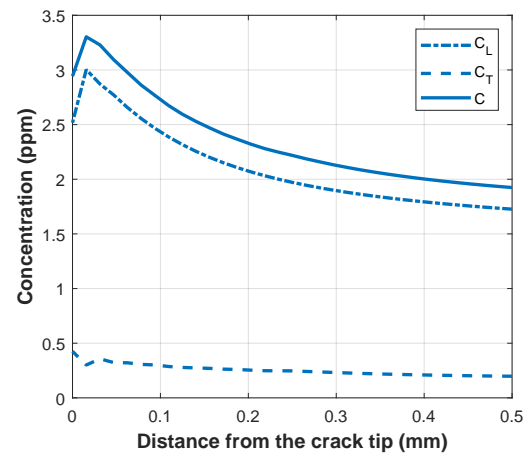

(a)

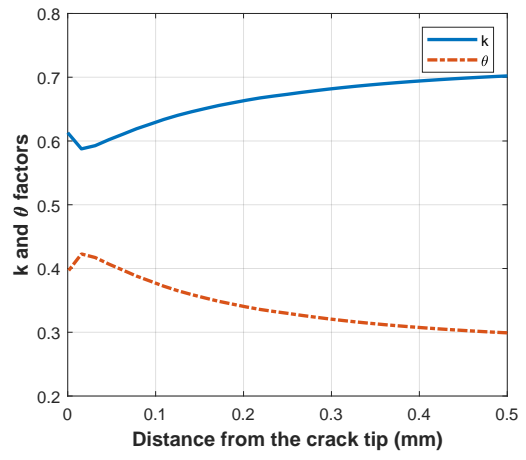

(c)
Fully coupled formulation

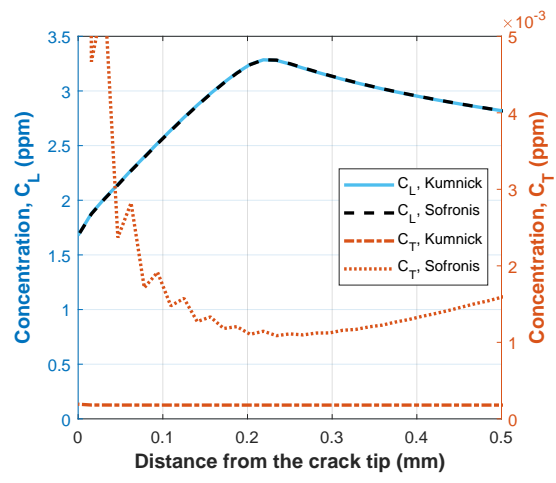

(b)

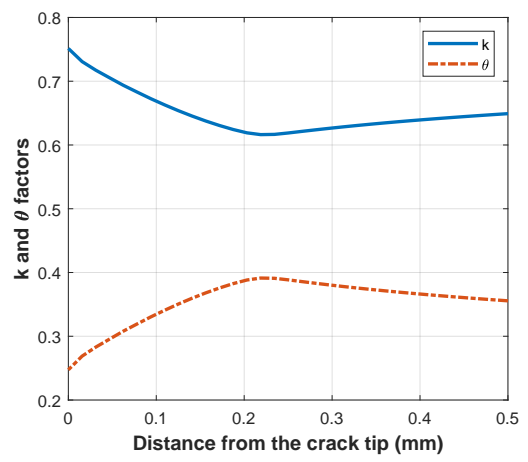

(d)

Figure 9: Main parameters of the simulation as a function of the distance from the crack tip, at the last frame before crack propagation: a) concentrations, weakly coupled formulation; b) concentrations, fully coupled formulation; c) coverage factor $\theta$ and decreasing factor $k$, weakly coupled formulation; d) coverage factor $\theta$ and decreasing factor $k$, fully coupled formulation. 
2. On the other hand, the estimation of $C_{T}$ with the fully coupled formulation provides values considerably lower than $C_{L}$, two orders of magnitude depending on the method ([23] or [25]), as visible in Figure $9 \mathrm{p}$. Since the $C_{L}$ is the dominant contribution, the method adopted to estimate the $C_{T}$ does not affect the results. Same considerations were found by [38], for clad steel pipes in API X60 steel. In this case, the total concentration, $C$, coincides with $C_{L}$ that shows a peak value of $3.3 \mathrm{ppm}$, as for the weakly coupled case, but located at $0.219 \mathrm{~mm}$ ahead of the crack tip.

3. In correspondence of the peak of the total concentration, the coverage factor $\theta$ exhibits the maximum value and the $k$ factor (used to decrease the cohesive strength) the minimum value (Figures 9r,d), as expected by definition.

\subsection{Sensitivity analysis}

This section reports the results of a sensitivity analysis performed on the model previously described implementing the fully coupled formulation. The main aim is to prove the capability of the model as a predictive tool for the estimation of the hydrogen effect on the mechanical properties of a steel. Indeed, this can provide useful information for the design and the assessment of components working in environments where the presence of hydrogen cannot be neglected. Two main parameters are considered for the sensitivity analysis, the diffusion coefficient, $D_{L}$, and the initial hydrogen concentration in the sample, $C_{0}$. Similar procedure was already adopted on the model implementing the weakly coupled formulation [39].

The parameter $D_{L}$, which drives the hydrogen flux, i. e. the velocity of the hydrogen atoms towards the crack, as shown in Eqs. 5 and 18, depends both on the lattice microstructure and the environmental conditions, as a function of the temperature. The experimental measurement of $D_{L}$ is challenging; it can be carried out by permeation and desorption curves. For the model, as described in the previous section, a default value of $D_{L}=1.0 \times 10^{-3} \mathrm{~mm}^{2} / \mathrm{s}$ was set, according to [40]. However, values between $1.0 \times 10^{-3}$ and $1.0 \times 10^{-6} \mathrm{~mm}^{2} / \mathrm{s}$ are considered in literature for the specific steel, AISI 4130 [39]. This range is used for the sensitivity analysis. For the features of the weakly coupled formulation, the variation of $D_{L}$ did not affect considerably the CTOD- $\Delta a$ curve [39]. On the other hand, Figure 10 reports interesting trends for the fully coupled implementation. Indeed, the CTOD- $\Delta a$ curve (Figure 10a) shows that $D_{L}$ influences the crack propagation; the higher is the $D_{L}$ value, the stronger is the hydrogen effect. In particular, considering a certain crack length $\Delta a$, the corresponding CTOD is up to $20 \%$ higher, when considering a decrease in $D_{L}$ of three orders of magnitude. Similarly, the peak of the total hydrogen concentration, $C$, ahead of the crack tip, which coincides with $C_{L}$, decreases from 3.28 to $1.78 \mathrm{ppm}(-46 \%)$, as shown in Figure $10 \mathrm{p}$. In addition, the peak moves from $219 \mu \mathrm{m}$ to $15.6 \mu \mathrm{m}$ (one-element length) from the crack tip, as well as the maximum and the minimum values of $k$ and $\theta$ factors, respectively, as visible in Figure 10 c.

The initial hydrogen concentration, $C_{0}$ is an environment-dependent parameter, related to the hydrogen content at the boundary of the steel structure. The default value assumed in the model for $C_{0}$ is $1.5 \mathrm{ppm}$. It is an average value derived from experimental measurements performed on the samples hydrogen charged with electro-chemical method and used for the experimental tests [20]. The sensitivity analysis was carried out considering a range of $C_{0}$ values between 0.5 


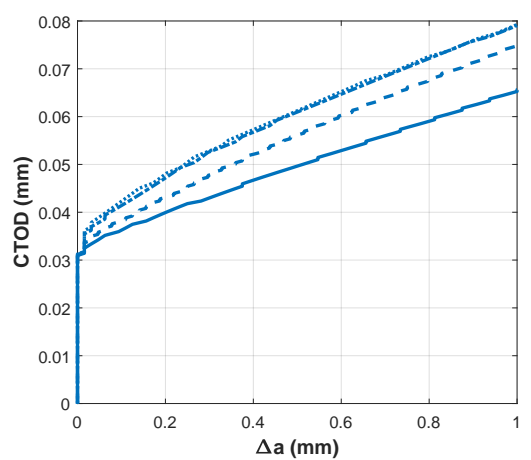

(a)

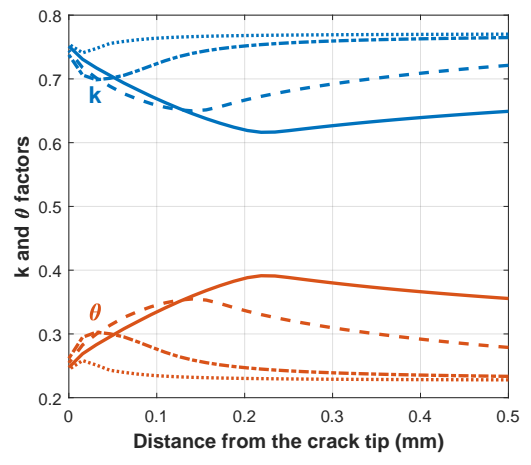

(c)

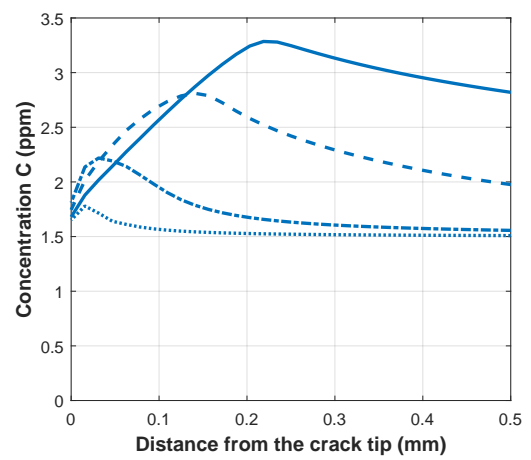

(b)

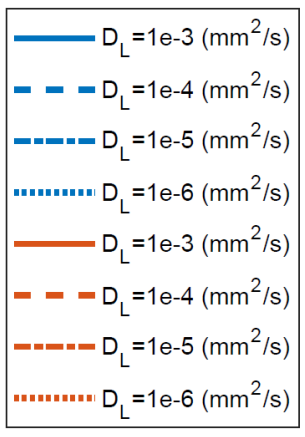

Figure 10: Results of the sensitivity analysis with the fully coupled formulation: variation of diffusivity, $D_{L}$. Plots of: (a) CTOD R-curve, (b) total hydrogen concentration $C$ vs distance from the crack tip, (c) $k$ and $\theta$ factors vs distance from the crack tip. 
and $3 \mathrm{ppm}$, typical values for industrial applications. Increasing the initial hydrogen content $C_{0}$, the crack growth rate progressively increases, as visible from the CTOD- $\Delta$ a curve in Figure 11a. However, while for lower values of $C_{0}$ the crack propagation increases considerably, for higher values of $C_{0}$ there is a kind of saturation effect, such that the propagation rate slightly changes. Indeed, for a fixed value of crack length, the CTOD values decrease of approximately $1 / 3$ passing from 0.5 to $1 \mathrm{ppm}$ and to an half from 0.5 to $3.0 \mathrm{ppm}$. The crack propagation initiation, instead, is strictly related to the TSL parameters, in particular to the final displacement $\delta_{F}$, according to the HEDE mechanism. This is a lower bound limiting the numerical result. Figure $11 \mathrm{p}$ shows the total hydrogen concentration in the specimen. This increases with the initial hydrogen content and the peak moves further from the crack tip (from $1.44 \mathrm{ppm}$ at $78 \mu \mathrm{m}$ from the crack tip, to $5.55 \mathrm{ppm}$ at $328 \mu \mathrm{m})$. Also in this case, the results of $k$ and $\theta$ factors are in agreement, with the total hydrogen concentration profile (Figure $11 \mathrm{c}$ ).

In conclusion, Figures 10 and 11 show that the variation of $D_{L}$ and $C_{0}$ induces similar effect. An increase of these parameters results in a higher crack growth rate and a higher hydrogen concentration at the crack tip with a peak value that moves far from it.

In general, the model in the fully coupled implementation results considerably sensitive to the variation of the input parameters. Depending on the values selected, the crack growth can be under or over-estimated up to $50 \%$. For this reason, particular attention has to be paid, if the numerical model is adopted to estimate the embrittling effect of hydrogen in a real structure. When experimental measurements of $D_{L}$ or reliable values of $C_{0}$ are not available, it is suggested to perform a sensitivity analysis, as the one herein described, in order to provide a range of mechanical responses of the structure to the applied loads.

\section{Conclusions}

The current paper presents a numerical implementation in a fully coupled form to simulate hydrogen embrittlement within the commercial software Abaqus. This is the complementary part of a previous paper [18] that deals with the weakly coupled formulation.

The fully coupled implementation accounts for the mutual interaction between the hydrogen diffusion and the stress-strain state, experienced during crack propagation. It relies on the analogy between mass diffusion and heat transfer equations. In particular, five user subroutines, which share variables through FORTRAN common blocks, are implemented in the coupled thermalstress analysis, available in the software, to calculate the total hydrogen concentration. The hydrogen content computed on the continuum elements is then transferred to the connected cohesive elements via the data structures implemented in the FORTRAN common blocks. A HEDE mechanism is simulated scaling the TSL, representative of the as-received material, based on the total hydrogen content.

It is worth noting that hydrogen flux depends on the hydrostatic stress gradient, as in Eq. (5), and therefore it is necessary to estimate this latter quantity at the Gauss points. For this purpose the hydrostatic stress, evaluated at the Gauss points, is first extrapolated to and averaged at the FE nodes: the gradient is then recovered by differentiating the nodal shape functions.

The paper provides a guideline to the methodology and also to the use of the code through a practical case: a FE cohesive zone model simulating the fracture toughness test of $\mathrm{C}(\mathrm{T})$ specimen 


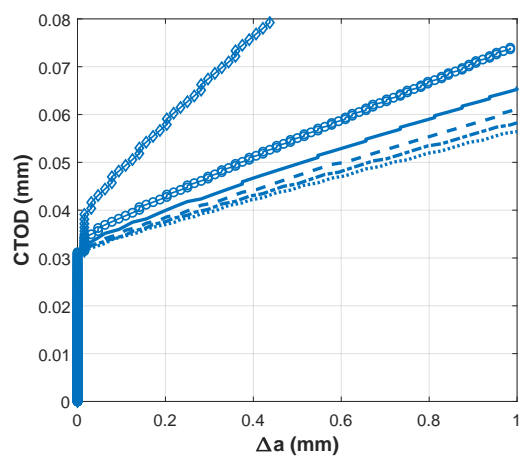

(a)

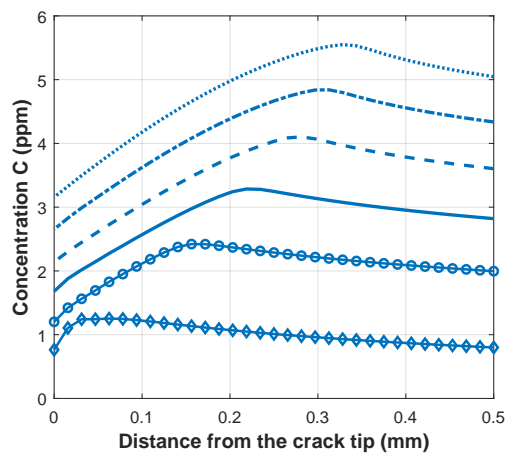

(b)
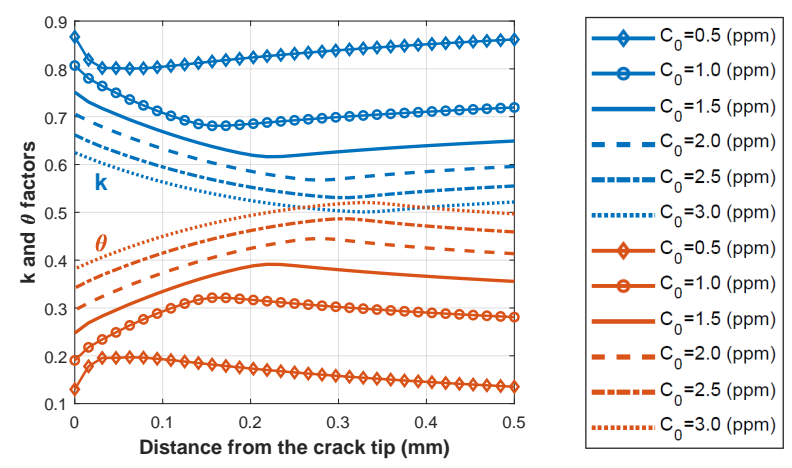

(c)

Figure 11: Results of the sensitivity analysis with the fully coupled formulation: variation of initial hydrogen concentration, $C_{0}$. Plots of: (a) CTOD- $\Delta a$ curve, (b) total hydrogen concentration $C$ vs distance from the crack tip, (c) $k$ (blue lines) and $\theta$ (red lines) factors vs distance from the crack tip. 
charged with hydrogen. The description of the model in the fully coupled formulation is enriched with a sensitivity analysis to prove the capability of the model as predictive tool in simulating hydrogen effect under different conditions.

As for the previous paper, the authors release the code under a permissive free software license: the codes as well as the examples herein presented are available in [19].

\section{Acknowledgment}

This research did not receive any specific grant from funding agencies in the public, commercial, or not-for-profit sectors.

[1] R. Gangloff, Hydrogen assisted cracking, Vol. 6, Elsevier Science, 2003, Ch. 02, pp. 31-101. doi:10.1016/ B0-08-043749-4/06134-6.

[2] D. Dugdale, Yielding of steel sheets containing slits, Journal of the Mechanics and Physics of Solids 8 (2) (1960) 100 - 104. doi:10.1016/0022-5096(60)90013-2.

[3] V. Olden, C. Thaulow, R. Johnsen, E. Ostbystby, T. Berstad, Application of hydrogen influenced cohesive laws in the prediction of hydrogen induced stress cracking in $25 \% \mathrm{Cr}$ duplex stainless steel, Engineering Fracture Mechanics 75 (8) (2008) 2333 - 2351. doi:10.1016/j.engfracmech.2007.09.003.

[4] V. Olden, A. Alvaro, O. M. Akselsen, Hydrogen diffusion and hydrogen influenced critical stress intensity in an API X70 pipeline steel welded joint - experiments and FE simulations, International Journal of Hydrogen Energy 37 (15) (2012) 11474 - 11486, hydrogen Enriched Methane. doi:10.1016/j.ijhydene.2012.05. 005

[5] A. Alvaro, V. Olden, O. M. Akselsen, 3D cohesive modelling of hydrogen embrittlement in the heat affected zone of an X70 pipeline steel, International Journal of Hydrogen Energy 38 (18) (2013) 7539 - 7549. doi: $10.1016 / j . i j h y d e n e .2013 .02 .146$

[6] A. Alvaro, V. Olden, O. M. Akselsen, 3D cohesive modelling of hydrogen embrittlement in the heat affected zone of an X70 pipeline steel - part II, International Journal of Hydrogen Energy 39 (7) (2014) 3528 - 3541. doi:10.1016/j.ijhydene.2013.12.097.

[7] L. Jemblie, V. Olden, O. M. Akselsen, A coupled diffusion and cohesive zone modelling approach for numerically assessing hydrogen embrittlement of steel structures, International Journal of Hydrogen Energy 42. doi:10.1016/j.ijhydene.2017.02.211

[8] W. Brocks, R. Falkenberg, I. Scheider, Coupling aspects in the simulation of hydrogen-induced stress-corrosion cracking, Procedia IUTAM 3 (2012) 11-24. doi:10.1016/j.piutam. 2012.03.002.

[9] A. Troiano, The role of hydrogen and other interstitials in the mechanical behaviour of metals, Transactions of American Society for Metals ASM 52 (1960) 54-80.

[10] H. Birnbaum, P. Sofronis, Hydrogen-enhanced localized plasticity-a mechanism for hydrogen-related fracture, Materials Science \& Engineering A: Structural Materials: Properties, Microstructure and Processing 176 (1-2) (1994) 191-202. doi:10.1016/0921-5093(94)90975-X.

[11] C. Moriconi, Modélisation de la propagation de fissure de fatigue assistée par l'hydrogène gazeux dans les matériaux métalliques, Ph.D. thesis, ISAE-ENSMA Ecole Nationale Supérieure de Mécanique et d'Aérotechique-Poitiers (2012).

[12] S. del Busto, C. Betegón, E. Martínez-Pañeda, A cohesive zone framework for environmentally assisted fatigue, Engineering Fracture Mechanics 185 (Supplement C) (2017) 210 - 226, XVIII International Colloquium Mechanical Fatigue of Metals. doi:10.1016/j.engfracmech.2017.05.021.

[13] O. Barrera, E. Tarleton, H. Tang, A. Cocks, Modelling the coupling between hydrogen diffusion and the mechanical behaviour of metals, Computational Materials Science 122 (Supplement C) (2016) 219 - 228. doi:10.1016/j.commatsci.2016.05.030

[14] N. Raykar, S. Maiti, R. S. Raman, Modelling of mode-i stable crack growth under hydrogen assisted stress corrosion cracking, Engineering Fracture Mechanics 78 (18) (2011) 3153 - 3165. doi:10.1016/j.engfracmech. 2011.07 .013 
[15] N. Raykar, S. Maiti, R. S. Raman, S. Aryan, Study of hydrogen concentration dependent growth of external annular crack in round tensile specimen using cohesive zone model, Engineering Fracture Mechanics 106 (2013) 49 - 66. doi:10.1016/j.engfracmech.2013.04.007.

[16] D. K. Singh, S. Maiti, T. K. Bhandakkar, R. S. Raman, Efficient approach for cohesive zone based threedimensional analysis of hydrogen-assisted cracking of a circumferentially notched round tensile specimen, International Journal of Hydrogen Energy 42 (24) (2017) 15943 - 15955. doi:10.1016/j.ijhydene.2017. 05.064

[17] D. K. Singh, S. Maiti, T. K. Bhandakkar, R. S. Raman, Cohesive zone based axisymmetric modelling of hydrogen-assisted cracking in a circumferentially notched tensile specimen, International Journal of Hydrogen Energy 43 (27) (2018) 12530 - 12542. doi:10.1016/j.ijhydene.2018.04.188

[18] G. Gobbi, C. Colombo, S. Miccoli, L. Vergani, A weakly coupled implementation of hydrogen embrittlement in FE analysis, Finite Elements in Analysis and Design 141 (2018) 17 - 25. doi:10.1016/j.finel.2017.11. 010.

[19] G. Gobbi, C. Colombo, S. Miccoli, L. Vergani, Polihydra/hydra: fully coupled implementation, v0.3.0 (Oct. 2018). doi:10.5281/zenodo.1478086.

[20] C. Colombo, G. Fumagalli, F. Bolzoni, G. Gobbi, L. Vergani, Fatigue behavior of hydrogen pre-charged low alloy Cr-Mo steel, International Journal of Fatigue 83 (2016) 2 - 9, from Microstructure to Design: Advances in Fatigue of Metals. doi:10.1016/j.ijfatigue.2015.06.002.

[21] P. Sofronis, R. McMeeking, Numerical analysis of hydrogen transport near a blunting crack tip, Journal of the Mechanics and Physics of Solids 37 (3) (1989) 317 - 350. doi:10.1016/0022-5096 (89) 90002-1.

[22] R. Oriani, The diffusion and trapping of hydrogen in steel, Acta Metallurgica 18 (1) (1970) 147 - 157. doi: 10.1016/0001-6160(70) 90078-7.

[23] A. Kumnick, H. Johnson, Deep trapping states for hydrogen in deformed iron, Acta Metallurgica 28 (1) (1980) 33 - 39. doi:10.1016/0001-6160(80)90038-3.

[24] A. Krom, R. Koers, A. Bakker, Hydrogen transport near a blunting crack tip, Journal of the Mechanics and Physics of Solids 47 (4) (1999) 971 - 992. doi : 10.1016/S0022-5096 (98) 00064-7.

[25] P. Sofronis, Y. Liang, N. Aravas, Hydrogen induced shear localization of the plastic flow in metals and alloys, European Journal of Mechanics - A/Solids 20 (6) (2001) 857 - 872. doi:10.1016/S0997-7538(01) 01179-2.

[26] S. Serebrinsky, E. Carter, M. Ortiz, A quantum-mechanically informed continuum model of hydrogen embrittlement, Journal of the Mechanics and Physics of Solids 52 (10) (2004) 2403 - 2430. doi:10.1016/j.jmps. 2004.02 .010

[27] Dassault Systèmes, Abaqus Analysis User's Guide (2016).

[28] I. Scheider, M. Pfuff, W. Dietzel, Simulation of hydrogen assisted stress corrosion cracking using the cohesive model, Engineering Fracture Mechanics 75 (15) (2008) 4283-4291. doi:10.1016/j.engfracmech. 2007. 10.002

[29] K.-H. Schwalbe, I. Scheider, A. Cornec, Guidelines for Applying Cohesive Models to the Damage Behaviour of Engineering Materials and Structures, Springer Berlin Heidelberg, 2013. doi : 10.1007/978-3-642-29494-5.

[30] R. A. Oriani, A mechanistic theory of hydrogen embrittlement of steels, Berichte der Bunsen-Gesellschaft für Physikalische Chemie 76 (8) (1972) 848-857. doi:10.1002/bbpc.19720760864

[31] C. D. Beachem, A new model for hydrogen-assisted cracking (hydrogen "embrittlement"), Metallurgical and Materials Transactions B 3 (2) (1972) 441-455. doi:10.1007/bf02642048.

[32] I. M. Robertson, The effect of hydrogen on dislocation dynamics, Engineering Fracture Mechanics 64 (5) (1999) 649-673. doi:10.1016/s0013-7944(99) 00094-6.

[33] J. Lufrano, P. Sofronis, H. Birnbaum, Modeling of hydrogen transport and elastically accommodated hydride formation near a crack tip, Journal of the Mechanics and Physics of Solids 44 (2) (1996) 179 - 205. doi: 10.1016/0022-5096(95) 00075-5.

[34] R. Matsumoto, S. Taketomi, S. Matsumoto, N. Miyazaki, Atomistic simulations of hydrogen embrittlement, International Journal of Hydrogen Energy 34 (23) (2009) 9576-9584. doi:10.1016/j.ijhydene.2009.09. 052

[35] M. Wen, S. Fukuyama, K. Yokogawa, Atomistic simulations of effect of hydrogen on kink-pair energetics of screw dislocations in bcc iron, Acta Materialia 51 (6) (2003) 1767-1773. doi:10.1016/s1359-6454(02) 
$00575-\mathrm{x}$

[36] J. Song, W. A. Curtin, Atomic mechanism and prediction of hydrogen embrittlement in iron, Nature Materials 12 (2) (2012) 145-151. doi:10.1038/nmat3479

[37] A. Taha, P. Sofronis, A micromechanics approach to the study of hydrogen transport and embrittlement, Engineering Fracture Mechanics 68 (6) (2001) 803 - 837. doi : 10.1016/S0013-7944(00)00126-0.

[38] L. Jemblie, V. Olden, P. Mainçon, O. Akselsen, Cohesive zone modelling of hydrogen induced cracking on the interface of clad steel pipes, International Journal of Hydrogen Energy 42 (47) (2017) 28622 - 28634. doi:10.1016/j.ijhydene.2017.09.051.

[39] G. Gobbi, C. Colombo, L. Vergani, Sensitivity analysis of a 2D cohesive model for hydrogen embrittlement of AISI4130, Engineering Fracture Mechanics 167 (2016) 101 - 111, SI:CRACK PATHS 2015. doi:10.1016/ j.engfracmech.2016.03.045

[40] R. L. S. Thomas, D. Li, R. P. Gangloff, J. R. Scully, Trap-governed hydrogen diffusivity and uptake capacity in ultrahigh-strength AERMET 100 steel, Metallurgical and Materials Transactions A 33 (7) (2002) 1991-2004. doi:10.1007/s11661-002-0032-6.

[41] W. P. Davey, Precision measurements of the lattice constants of twelve common metals, Physical Review 25 (6) (1925) 753-761. doi:10.1103/physrev.25.753

[42] W. F. Gale, T. C. Totemeier (Eds.), Smithells Metals Reference Book, 8th Ed., Elsevier, 2004.

[43] P. J. Mohr, D. B. Newell, B. N. Taylor, CODATA recommended values of the fundamental physical constants: 2014*, Reviews of Modern Physics 88 (2016) 035009. arXiv: 1507.07956, doi:10.1103/RevModPhys . 88. 035009 


\section{AppendixA. Code organisation}

The implementation of the present formulation along with relevant files (mesh, data, etc.) has been archived in [19]. That repository includes also step-by-step instructions for running the code and reproducing the study cases presented in this paper. However a brief overview is included in this appendix for the sake of clarity and completeness; moreover Table A.2 contains the values of all relevant quantities used for running the simulations.

Three main steps are required to run a fully coupled analysis:

1. Preparation of the Abaqus model (*. inp files).

2. Preparation of auxiliary files read by the user subroutines (*.jac and *. map files)

3. Abaqus analysis run.

Step 1. is detailed in [18, 19]; a Python program is provided that generates the Abaqus cards for the TSL of Fig. 5 as a function of $\delta_{0}, \delta_{1}, \delta_{F}$, and $t_{n}^{0}$.

Step 2. requires an Abaqus data check run on the input files of step 1. Abaqus Python programs are provided that read the output database .odb file and generate two text files: a . jac with precomputed Jacobian matrix entries at the integration points of the solid elements (useful for the stress gradient computation) and a .map file, which defines the mapping between the cohesive element numbers and the adjacent solid elements.

Once these files are generated, the fully coupled analysis can be started; this analysis requires five user subroutines. At the core of the procedure is the UMATHT subroutine, which implements the mass diffusion problem. Quantities needed in this analysis are provided through a common block by the other user subroutines, URDFIL, UVARM, and USDFLD. The latter two subroutines, as outlined in [18] also compute and map to the cohesive elements quantities related to the TSL formulation, Eqs. 20) and (21).

The interaction of the provided user subroutines with the Abaqus non-linear solution procedure loop is outlined in the flowchart of fig. A.12. 


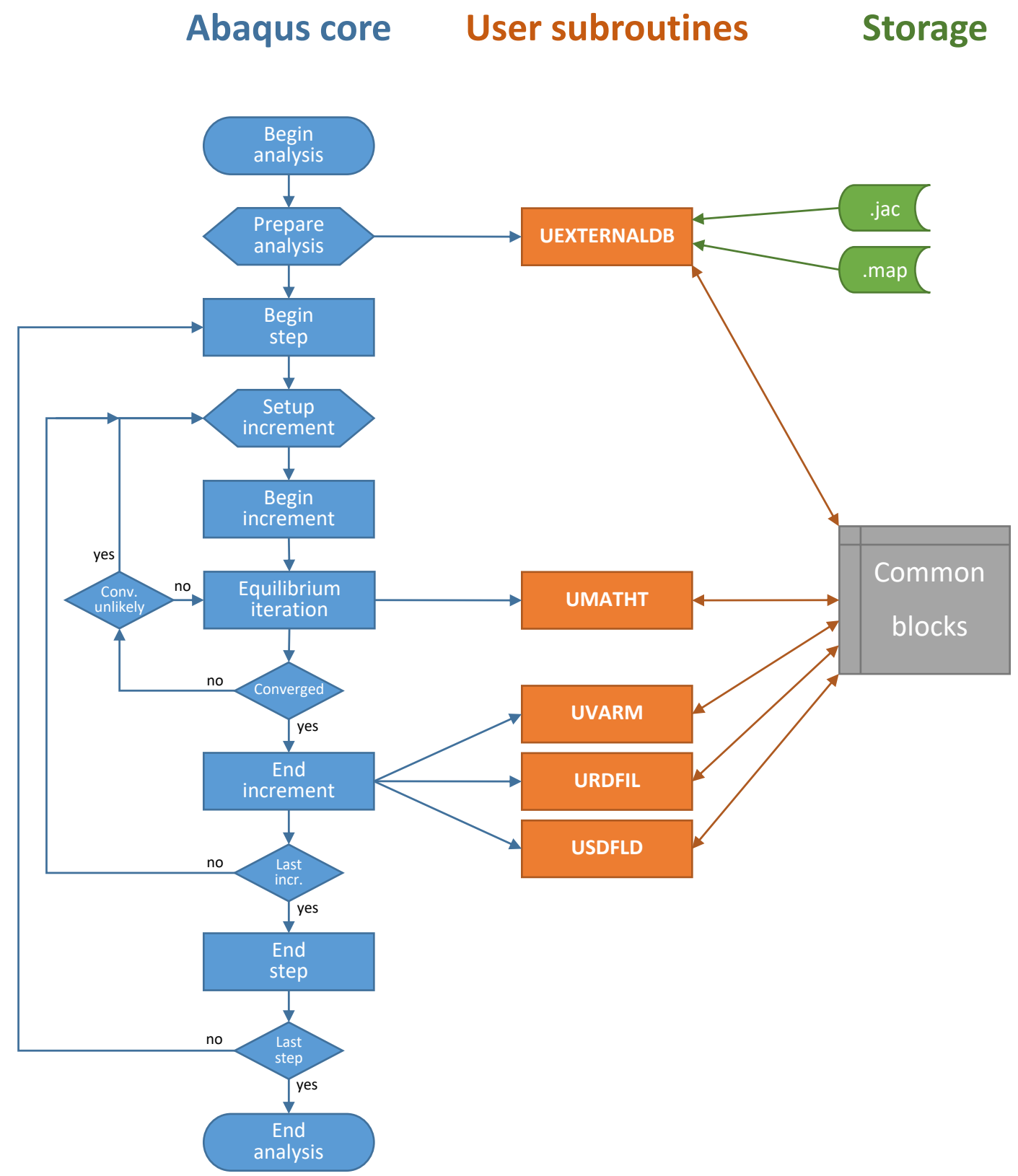

Figure A.12: User subroutine call flowchart. 
Table A.2: Values of input model parameters

\begin{tabular}{lcc}
\hline Variable & Value & Reference \\
\hline $\bar{a}$ & $2.86 \times 10^{-10} \mathrm{~m}$ & {$[41]$} \\
$A_{r}$ & $55.8 \times 10^{-3} \mathrm{~kg} / \mathrm{mol}$ & {$[42]$} \\
$C_{0}$ & $1.5 \mathrm{ppm}$ & {$[20]$} \\
$D_{\mathrm{L}}$ & $1.0 \times 10^{-9} \mathrm{~m}^{2} / \mathrm{s}$ & {$[40]$} \\
$N_{\mathrm{A}}$ & $6.0221 / \mathrm{mol}$ & {$[43]$} \\
$R$ & $8.3145 \mathrm{~J} /(\mathrm{mol} \mathrm{K})$ & {$[43]$} \\
$T$ & $296.15 \mathrm{~K}$ & {$[20]$} \\
$t_{n}^{0}$ & $2.6 \times 10^{9} \mathrm{~Pa}$ & {$[18,[19]$} \\
$V_{\mathrm{H}}$ & $2 \times 10^{-6} \mathrm{~m}^{3} / \mathrm{mol}$ & {$[26]$} \\
$\beta$ & 6 & {$[24]$} \\
$\gamma$ & $2 \times 10^{16} \mathrm{~m} / \mathrm{m}^{3}$ & {$[25]$} \\
$\delta_{0}$ & $7.5 \times 10^{-7} \mathrm{~m}$ & {$[18,[19]$} \\
$\delta_{1}$ & $9.75 \times 10^{-6} \mathrm{~m}$ & {$[18,19]$} \\
$\delta_{F}$ & $1.57 \times 10^{-5} \mathrm{~m}$ & {$[18,19]$} \\
$\Delta g_{b}$ & $3 \times 10^{4} \mathrm{~J} / \mathrm{mol}$ & {$[26]$} \\
$\Delta W_{B}$ & $-6 \times 10^{4} \mathrm{~J} / \mathrm{mol}$ & {$[23]$} \\
$\rho$ & $7.87 \times 10^{3} \mathrm{~kg} / \mathrm{m}^{3}$ & {$[24]$} \\
$\bar{\rho}_{0}$ & $1 \times 10^{10} \mathrm{~m} / \mathrm{m}^{3}$ & {$[25]$} \\
$\bar{\rho}_{1}$ & $1 \times 10^{16} \mathrm{~m} / \mathrm{m}^{3}$ & {$[25]$} \\
\hline
\end{tabular}

